\title{
QUEEN'S
QNEIVERSITY
BELFAST
}

\section{Tailoring the nonlinear frequency coupling between odd harmonics for the optimisation of charged particle dynamics in capacitively coupled oxygen plasmas}

Gibson, A. R., Greb, A., Graham, W. G., \& Gans, T. (2015). Tailoring the nonlinear frequency coupling between odd harmonics for the optimisation of charged particle dynamics in capacitively coupled oxygen plasmas. Applied Physics Letters, 106(5), [054102]. https://doi.org/10.1063/1.4907567

Published in:

Applied Physics Letters

Document Version:

Publisher's PDF, also known as Version of record

Queen's University Belfast - Research Portal:

Link to publication record in Queen's University Belfast Research Portal

Publisher rights

Copyright 2015 AIP Publishing LLC

\section{General rights}

Copyright for the publications made accessible via the Queen's University Belfast Research Portal is retained by the author(s) and / or other copyright owners and it is a condition of accessing these publications that users recognise and abide by the legal requirements associated with these rights.

Take down policy

The Research Portal is Queen's institutional repository that provides access to Queen's research output. Every effort has been made to ensure that content in the Research Portal does not infringe any person's rights, or applicable UK laws. If you discover content in the Research Portal that you believe breaches copyright or violates any law, please contact openaccess@qub.ac.uk. 


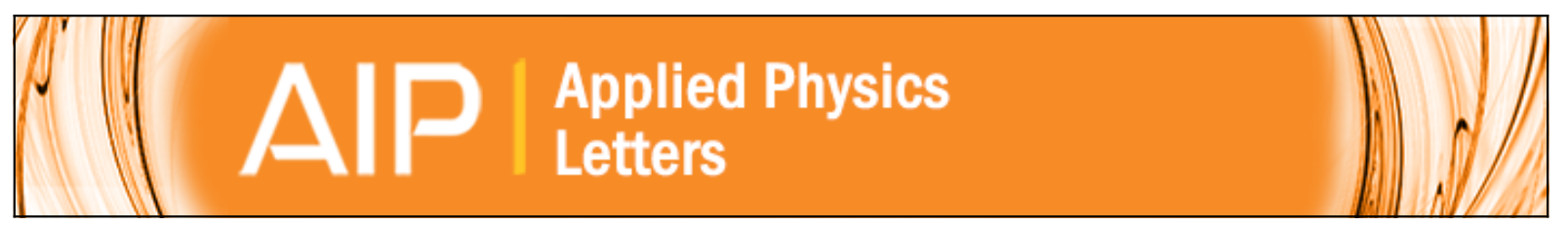

Tailoring the nonlinear frequency coupling between odd harmonics for the optimisation of charged particle dynamics in capacitively coupled oxygen plasmas

A. R. Gibson, A. Greb, W. G. Graham, and T. Gans

Citation: Applied Physics Letters 106, 054102 (2015); doi: 10.1063/1.4907567

View online: http://dx.doi.org/10.1063/1.4907567

View Table of Contents: http://scitation.aip.org/content/aip/journal/apl/106/5?ver=pdfcov

Published by the AIP Publishing

\section{Articles you may be interested in}

Kinetic simulation of capacitively coupled plasmas driven by trapezoidal asymmetric voltage pulses

J. Appl. Phys. 115, 233302 (2014); 10.1063/1.4884017

Narrow gap electronegative capacitive discharges

Phys. Plasmas 20, 101603 (2013); 10.1063/1.4823459

Simulation study of wave phenomena from the sheath region in single frequency capacitively coupled plasma discharges; field reversals and ion reflection

Phys. Plasmas 20, 073507 (2013); 10.1063/1.4816952

Test particle simulation of the role of ballistic electrons in hybrid dc/rf capacitively coupled C F 4 plasmas

J. Vac. Sci. Technol. A 27, 287 (2009); 10.1116/1.3072922

Theory and particle simulation of nonlinear double layers in a magnetized plasma

Phys. Plasmas 14, 052301 (2007); 10.1063/1.2722291

You don't

still use this

cell phone

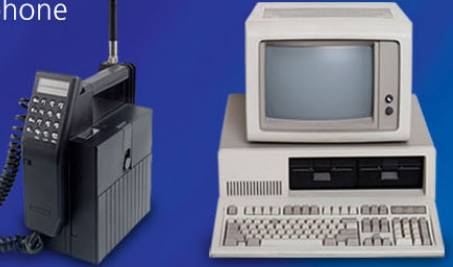

Why are you still using an AFM designed in the 80 's?
It is time to upgrade your AFM

Minimum \$20,000 trade-in discount for purchases before August 31st

Asylum Research is today's technology leader in AFM 


\title{
Tailoring the nonlinear frequency coupling between odd harmonics for the optimisation of charged particle dynamics in capacitively coupled oxygen plasmas
}

\author{
A. R. Gibson, ${ }^{1,2, a)}$ A. Greb, ${ }^{2}$ W. G. Graham, ${ }^{1}$ and T. Gans ${ }^{2}$ \\ ${ }^{1}$ Centre for Plasma Physics, Queen's University Belfast, University Road, Belfast BT7 1NN, United Kingdom \\ ${ }^{2}$ York Plasma Institute, Department of Physics, University of York, Heslington, York YO10 5DQ, United Kingdom
}

(Received 16 November 2014; accepted 19 January 2015; published online 2 February 2015)

\begin{abstract}
The influence of nonlinear frequency coupling in an oxygen plasma excited by two odd harmonics at moderate pressure is investigated using a numerical model. Through variations in the voltage ratio and phase shift between the frequency components changes in ionization dynamics and sheath voltages are demonstrated. Furthermore, a regime in which the voltage drop across the plasma sheath is minimised is identified. This regime provides a significantly higher ion flux than a single frequency discharge driven by the lower of the two frequencies alone. These operating parameters have potential to be exploited for plasma processes requiring low ion bombardment energies but high ion fluxes. (C) 2015 AIP Publishing LLC. [http://dx.doi.org/10.1063/1.4907567]
\end{abstract}

Non-equilibrium capacitively coupled plasma (CCP) sources produced at low to intermediate pressures are widely used in various industries, in particular, for etching and deposition processes in nanoscale semiconductor fabrication. In this context, the control of plasma properties such as the ion bombardment energy (IBE) and ion flux to the substrate is crucially important to achieve the required material characteristics. Generally, high ion fluxes are preferred for most applications as this is one of the main quantities in determining the rate of the particular process. However, the required IBE is largely determined by the process of interest.

Various strategies have arisen to control ion fluxes and IBEs at the substrate. Some of the most prominent of these have focussed on driving the plasma using a voltage waveform composed of multiple frequencies. ${ }^{1-6}$ Conventionally, this involves exciting a plasma with two significantly differing frequencies, ${ }^{1,2}$ with the phase between them being generally unspecified. In principle, this technique can allow for separate control of ion flux and IBE, with the high frequency controlling the former, and the low frequency the latter. Under these conditions, the two frequencies may be described as functionally separated. ${ }^{2}$ However, if very high frequencies are used in these systems electromagnetic effects begin to become important, ${ }^{6-8}$ these contribute to non-uniformities in the plasma and therefore inhomogeneities in the plasma process across the substrate. Such effects become particularly pronounced in large area plasma reactors, such as those used for thin film deposition in solar cell manufacturing. ${ }^{8}$

When the frequencies used are lower and less separated in frequency space the nonlinear coupling between them can also limit plasma control. ${ }^{4,9,10}$ In this regime, the two frequencies are no longer functionally separated. However, when properly controlled, this nonlinear coupling may be advantageous for plasma processing. An example of this is the electrical asymmetry effect (EAE) which utilises a driving waveform composed of two or more consecutive harmonics. ${ }^{5,11-15}$ The result is an electrically asymmetric

${ }^{\text {a)} E l e c t r o n i c ~ m a i l: ~ a g i b s o n 17 @ q u b . a c . u k ~}$ waveform where a dc bias is produced, even in a geometrically symmetric system. The value of this bias is then adjusted by either changing the number of harmonics used or the phase shift between them. However, the degree to which the dc bias voltage may be varied using this technique is reduced under certain conditions, such as high electronegativity ${ }^{11}$ or in gases where field reversal ionization is significant. ${ }^{14}$ Under such conditions, other methods of utilising nonlinear frequency coupling to gain greater control of plasma dynamics warrant further investigation. For example, the use of odd harmonics, where no electrical asymmetry is produced has been shown to provide advantageous control of plasma properties at atmospheric pressure. ${ }^{16,17}$

Here, the nonlinear coupling between odd harmonics is investigated for the control of ion fluxes and IBEs at lower pressures. A benchmarked ${ }^{18,19}$ one-dimensional (1D) fluid simulation of a CCP in oxygen with a semi-kinetic treatment of plasma electrons and energy dependent ion mobilities is used. Full details of the model are given in Ref. 19. The modelled system is assumed to be symmetric, with the electrode gap $(25 \mathrm{~mm})$ being much smaller than the electrode diameter, hence justifying a 1D treatment. In the model, one electrode is grounded while the other is driven by the voltage waveform shown in Eq. (1)

$$
V=V_{l f} \cos \left(2 \pi f_{l f} t\right)+V_{h f} \cos \left(2 \pi f_{h f} t+\theta\right) .
$$

Here, $V_{l f}, V_{h f}, f_{l f}$, and $f_{h f}$ are the amplitudes and frequencies of the low and high frequency components, denoted by (lf) and (hf), respectively. $\theta$ is the specified phase shift between the two frequency components. In this study the low and high frequencies are chosen to be $13.56 \mathrm{MHz}$ and its third harmonic $40.68 \mathrm{MHz}$, respectively. These are chosen as they are close enough in frequency space to exhibit significant frequency coupling ${ }^{17}$ without producing an electrically asymmetric plasma. Unlike $13.56 \mathrm{MHz}$, which is a frequency commonly used in industrial processing applications, there are indications that a single frequency $40.68 \mathrm{MHz}$ discharge would induce unacceptable non-uniformities across the substrate 
in very large scale reactors. ${ }^{8}$ However, Hrunski et al. ${ }^{8}$ have found in the case of $13.56 \mathrm{MHz}$ and $27.12 \mathrm{MHz}$ that even small $13.56 \mathrm{MHz}$ contributions to the overall waveform can provide much better uniformity than a $27.12 \mathrm{MHz}$ single frequency plasma. In this context, it is likely that small hf component contributions to the overall waveform will also be favourable for such applications using the odd harmonic approach. In this study we focus on the ideal frequency component ratios and phase shifts for optimising ion fluxes and IBEs at the substrate.

As a commonly used gas in industry, oxygen serves as a useful test scenario for the investigation of nonlinear frequency coupling. It is weakly electronegative under the investigated conditions and as such gives an indication of how frequency coupling will behave in other more strongly electronegative gases also used in industry. Thus, molecular oxygen $\left(\mathrm{O}_{2}\right)$ is incorporated into the model as the background gas at $40 \mathrm{~Pa}$ and $300 \mathrm{~K}$ and is assumed to remain at a constant density. The plasma chemistry is purposely simple and takes account of electrons (e), positive molecular oxygen ions $\left(\mathrm{O}_{2}^{+}\right)$and negative atomic oxygen ions $\left(\mathrm{O}^{-}\right)$using 8 reactions. Secondary electron emission is neglected in order to exclude any effect of secondary electrons on frequency coupling, however under the low voltage conditions investigated here this is not expected to significantly affect the observed trends. Singlet delta oxygen $\left(\mathrm{O}_{2}\left({ }^{1} \Delta\right)\right)$, an important metastable molecule in oxygen discharges, ${ }^{20}$ is included and assumed to be $\approx 1 / 6$ of the background gas density, based on the work of Bronold et al. ${ }^{21}$ For electrons, the nonMaxwellian electron energy distribution function (EEDF) is calculated in advance using the OD Boltzmann equation solver BOLSIG $+{ }^{22}$ The resulting electron transport coefficients and electron impact reaction rates are then incorporated into the 1D fluid model as lookup tables.

Initially, the change in plasma properties are investigated as a function of the fraction of each frequency contributing to the overall waveform, throughout this variation $\theta=0^{\circ}$. The resulting time and space resolved electron impact ionization rate of $\mathrm{O}_{2}$ is shown in Figures 1(a)-1(c) for (a) $V_{l f}=200 \mathrm{~V}, V_{h f}=0 \mathrm{~V}$ (lf only), (b) $V_{l f}=V_{h f}=100 \mathrm{~V}$ (dual frequency or df), and (c) $V_{l f}=0 \mathrm{~V}, V_{h f}=200 \mathrm{~V}$ (hf only). The powered electrode is at $0 \mathrm{~mm}$ and the grounded electrode at $25 \mathrm{~mm}$. The dashed line represents the momentary sheath edge position, calculated assuming an equivalent sharp electron density step. ${ }^{23}$ Figure $1(\mathrm{~d})$ shows the voltage drop across the powered sheath $\left(V_{s, p o w}\right)$ for the conditions shown in Figures 1(a)-1(c). It is observed here that as the contribution of the hf component to the overall waveform increases, the ionization rate increases with it as reflected in the differing scales of Figures 1(a)-1(c).

The origin of the ionization structures in Figures 1(a)-1(c) can be explained by comparison with Figure 1(d). For lf only excitation $V_{s, p o w}$ increases and decreases once per If cycle, corresponding to an increase and decrease in the size of the plasma sheath. As the electronegativity of the investigated oxygen plasma is relatively low $(\approx 1)$, the dominant electron heating is caused by the expansion of the plasma sheath. ${ }^{18}$ This heating causes the ionization observed in Figure 1 (a) at $\approx 25$ ns at the powered sheath, and the corresponding structure at $\approx 62 \mathrm{~ns}$ at the grounded sheath. For hf

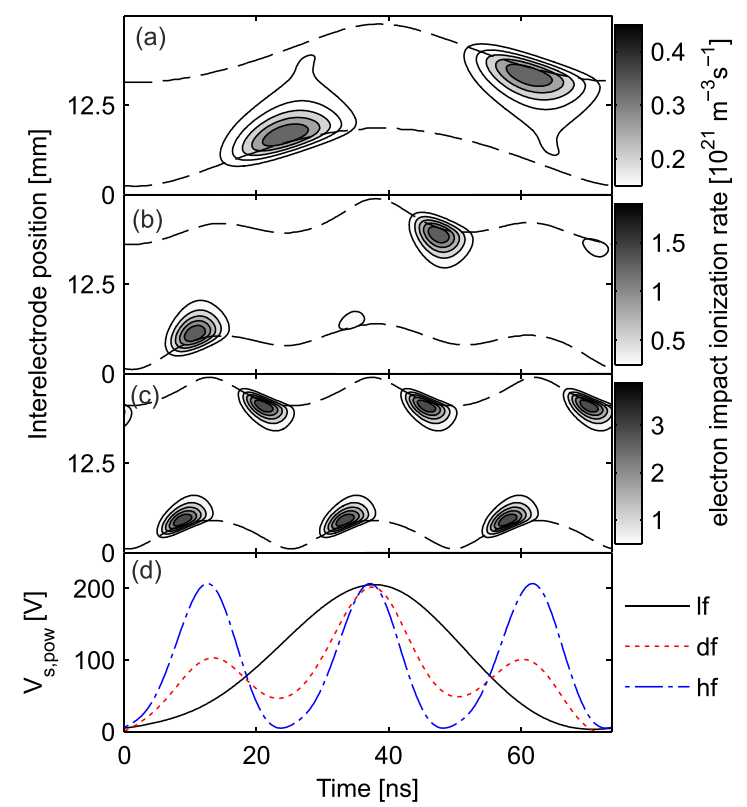

FIG. 1. (a) Time and space resolved electron impact ionization rate of $\mathrm{O}_{2}$ for three different frequency ratios: (a) $V_{l f}=200 \mathrm{~V}, V_{h f}=0 \mathrm{~V}$ (lf only), (b) $V_{l f}=V_{h f}=100 \mathrm{~V}$ (df), and (c) $V_{l f}=0 \mathrm{~V}, V_{h f}=200 \mathrm{~V}$ (hf only). (d) Voltage drop over the powered electrode sheath $\left(V_{s, p o w}\right)$ as a function of time for the same conditions as (a)-(c).

only excitation, $V_{s, p o w}$ increases and decreases three times per lf cycle because three hf cycles occur inside one lf cycle. This results in a much faster change in $V_{s, p o w}$ on each sheath expansion, in a given time, than for If only excitation. The result of this is three, more intense sheath expansion ionization structures at the powered electrode, and three equivalent structures at the grounded electrode.

The superposition of both frequencies leads to a more complex sheath structure than one frequency alone, as shown in Figure 1(d). For df, $V_{s, p o w}$ initially increases to $100 \mathrm{~V}$ from $0 \mathrm{~ns}$ to $14 \mathrm{~ns}$ before decreasing to $50 \mathrm{~V}$ from $14 \mathrm{~ns}$ to $24 \mathrm{~ns}$ and increasing again to its overall maximum at $37 \mathrm{~ns}$. The result of this sheath structure is two visible ionization rate structures of different intensities at the powered electrode, and two corresponding structures at the grounded electrode. The differing intensity of the sheath expansion structures at each electrode is a phenomenon unique to multiple frequency excitation and can be explained by the changing velocity of the plasma sheath edge. The most intense ionization rate structure at the powered electrode occurs at approximately $8 \mathrm{~ns}$, followed by a less intense structure at $33 \mathrm{~ns}$. The difference in intensity can be explained as follows: in general, the electrons are heated by the accelerating sheath edge with the heating and ionization rate scaling with its velocity. This velocity is dependent upon the local ion density at the sheath edge. For the same voltage change, the sheath travels further in a given time where the ion density is lowest. At $8 \mathrm{~ns}$, the plasma sheath edge is close to the electrodes, where the ion density is low. Therefore, the plasma sheath edge velocity is at its highest value for a given voltage change, causing the highest possible ionization rate. At $33 \mathrm{~ns}$, the sheath edge is closer to the discharge centre, where the ion densities are higher leading to a lower sheath velocity and ionization rate. The varying ion density at the sheath edge thus leads to 
the increased ionization rate at $8 \mathrm{~ns}$ compared to that at $33 \mathrm{~ns}$, despite the fact that the gradient in the $V_{s, p o w}$ at $33 \mathrm{~ns}$ is greater as shown in Figure 1(d).

In order to further control electron heating and sheath voltages, the phase shift $\theta$ between the two frequencies may be varied. Figures 2(a) and 2(b) show the resulting time and space resolved electron impact ionization of $\mathrm{O}_{2}$, with $V_{l f}=V_{h f}=100 \mathrm{~V}$ for (a) $\theta=0^{\circ}$ and (b) $\theta=180^{\circ}$. On the introduction of a $180^{\circ}$ phase shift to the hf component the electron heating can be confined in time to produce more intense ionization structures at $21 \mathrm{~ns}$ at the powered electrode and $58 \mathrm{~ns}$ at the grounded electrode. Furthermore the time averaged ionization rate is also enhanced by approximately $11 \%$. The reasons for these differences can again be explained by examining $V_{s, p o w}$, as shown in Figure 2(c). The peak in ionization for $\theta=180^{\circ}$ at $21 \mathrm{~ns}$ can be attributed to the sheath expansion from $10 \mathrm{~ns}$ to $25 \mathrm{~ns}$. Here, the sheath voltage increases in one expansion from its minimum to its maximum, analogous to the single frequency case. The increase in intensity of the ionization rate structure is a result of the high gradient in $V_{\text {s.pow }}$ and the lower ion density in this region, as discussed before. However, in this case the maximum sheath voltage drop has been decreased by applying the phase shift. This occurs because of destructive interference between the two frequencies which results in a decrease in the overall waveform amplitude.

The observation that the overall ionization rate increases, while the maximum sheath voltage drop decreases is interesting from an application perspective, in particular, for plasma processes requiring high ion fluxes to the substrate and low ion energies. To determine an optimum operating regime for application of this technique in such processes, the average voltage drop over the powered sheath $\left(\bar{V}_{s, p o w}\right)$ and positive ion flux at the powered electrode $\left(\Gamma_{+}\right)$are shown in Figures 3(a) and 3(b), respectively. These are visualised as contour plots where the ordinates show the phase shift, $\theta$, and the abscissa show the percentage hf component contributing to the overall waveform. At all points $V_{l f}+V_{h f}=200 \mathrm{~V}$.

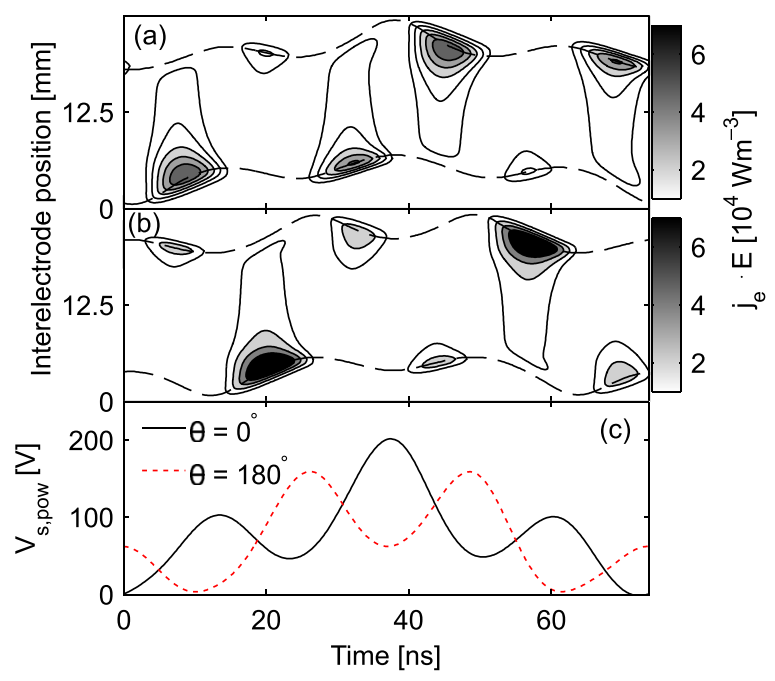

FIG. 2. Time and space resolved electron impact ionization of $\mathrm{O}_{2}$ for (a) $V_{l f}=V_{h f}=100 \mathrm{~V}$ and $\theta=0^{\circ}$ and (b) $V_{l f}=V_{h f}=100 \mathrm{~V}$ and $\theta=180^{\circ}$. (c) Voltage drop over the powered electrode sheath $\left(V_{s, p o w}\right)$ as a function of time for the same conditions as (a) and (b).
Figure 3(a) shows a minimum in the average sheath voltage drop at $40 \% \mathrm{hf}$ component and $\theta=180^{\circ}$ which is approximately $25 \%$ less than the equivalent value for lf only excitation. This is an obvious regime for processes requiring low IBEs at the substrate. In particular, the maximum energy of the ion energy distribution function is largely determined by the average voltage drop across the plasma sheath, as such it is expected that this regime will offer lower maximum ion bombardment energy. In Figure 3(b), it is observed that $\Gamma_{+}$increases with increasing hf component as expected due to more efficient ionization at higher hf contributions as discussed previously. Therefore, with regard to high ion fluxes the best operating parameters are high hf components, with the phase shift playing little role in changing the plasma density in this regime. The caveat to this, as discussed earlier, is that at very high hf contributions in very large scale reactors, electromagnetic effects will likely introduce plasma non-uniformities, unacceptable from a processing perspective. So in such applications this regime is likely to be unattractive. However, even with smaller hf contributions to the overall waveform the plasma density can be increased significantly as compared to If only excitation. For instance at $40 \%$ hf component and $\theta=180^{\circ}$, where the sheath voltage is at its minimum value, $\Gamma_{+}$is enhanced by a factor of $\approx 2.1$ compared to lf only excitation. In this context such a regime may prove to be attractive for very large scale manufacturing processes.

To further emphasise the potential of this technique, Figure 4 shows the average voltage drop across the powered electrode sheath plotted against the average ion flux at the powered electrode for three different parameter variations: (i) lf only with $V_{l f}$ increasing from $200 \mathrm{~V}$ to $400 \mathrm{~V}$, (ii) $V_{l f}+V_{h f}$ $=200 \mathrm{~V}, \theta=0^{\circ}$, and (iii) $V_{l f}+V_{h f}=200 \mathrm{~V}, \theta=180^{\circ}$, both with the percentage $\mathrm{hf}$ increasing incrementally along the abscissa. For (ii) and (iii) the maximum hf percentage shown is

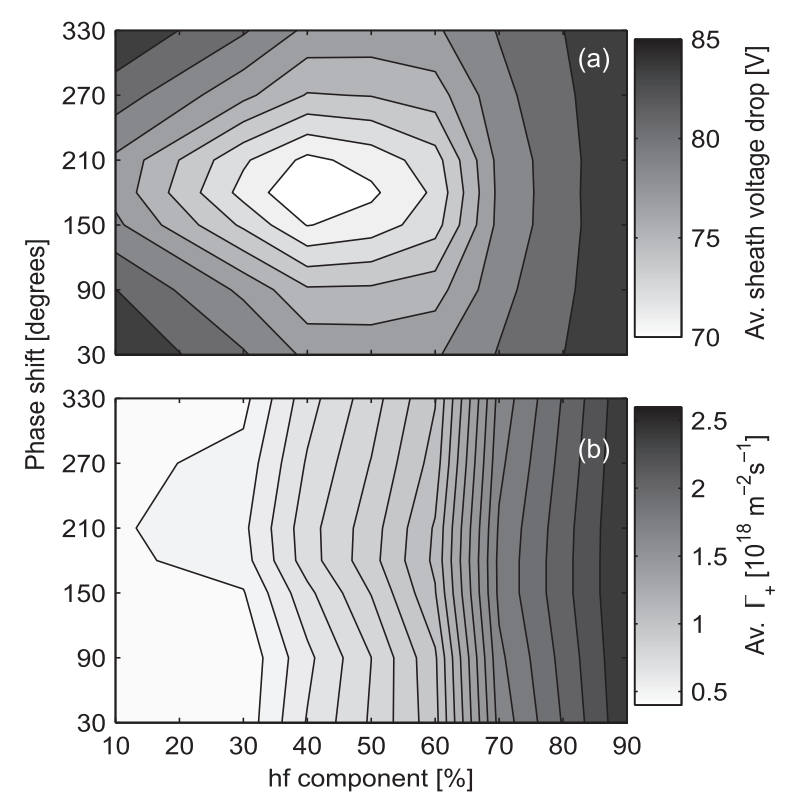

FIG. 3. Contour plots of (a) average sheath voltage drop and (b) average ion flux $\left(\Gamma_{+}\right)$at the powered electrode for $V_{l f}+V_{h f}=200 \mathrm{~V}$, the abscissa show percentage $\mathrm{hf}$ component and the ordinates show the phase shift between the components. 


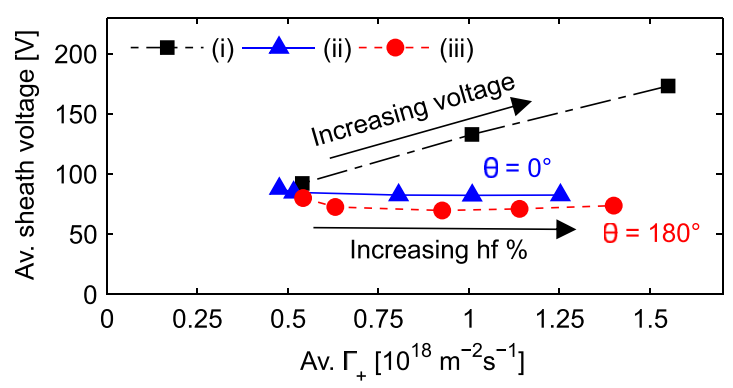

FIG. 4. Average sheath voltage drop plotted against average $\Gamma_{+}$at the powered electrode for (i) lf only with $V_{l f}$ increasing from $200 \mathrm{~V}$ to $400 \mathrm{~V}$, (ii) $V_{l f}+V_{h f}=200 \mathrm{~V}, \theta=0^{\circ}$, and (iii) $V_{l f}+V_{h f}=200 \mathrm{~V}, \theta=180^{\circ}$, both with the percentage hf increasing along the abscissa. For (ii) and (iii) the maximum hf percentage shown is $60 \%$.

$60 \%$, this is the highest hf component deemed likely to be acceptable from the point of view of uniformities across the substrate, and is motivated by the work of Hrunski et al. ${ }^{8}$ Here, the advantage of introducing the hf component is made more clear. Comparing (i) and (ii) it is observed that much higher sheath voltages are required for lf only to sustain the same ion flux as can be attained by mixing hf and if components. It is also notable that (iii) is shifted to lower average sheath voltages and higher ion fluxes compared to (ii) as a result of the discussed advantages of introducing a phase shift between the two frequency components.

In conclusion, the influence of nonlinear frequency coupling in a weakly electronegative capacitively coupled oxygen plasma has been demonstrated and explained. The mixing of the two frequencies with a constant phase shift of $\theta=0^{\circ}$ has been shown to increase ionization and modulate the time resolved sheath voltage. The introduction of a phase shift can further influence the ionization rate and sheath voltage. By comparison with standard single frequency excitation, it has been shown that even the introduction of a small hf percentage to the overall waveform with constant voltage contributions from the two frequencies can potentially offer enhanced conditions for plasma processing applications requiring low IBEs and high ion fluxes.
The authors acknowledge funding through a Northern Ireland Department of Employment and Learning (NI DEL) studentship and UK Engineering and Physical Sciences Research Council (EPSRC) Manufacturing Grant (EP/K018388/1).

${ }^{1}$ H. Goto, H.-D. Löwe, and T. Ohmi, IEEE Trans. Semicond. Manuf. 6, 58 (1993).

${ }^{2}$ T. Kitajima, Y. Takeo, Z. L. Petrović, and T. Makabe, Appl. Phys. Lett. 77, 489 (2000).

${ }^{3}$ S. B. Wang and A. E. Wendt, J. Appl. Phys. 88, 643 (2000).

${ }^{4}$ T. Gans, J. Schulze, D. O'Connell, U. Czarnetzki, R. Faulkner, A. R. Ellingboe, and M. M. Turner, Appl. Phys. Lett. 89, 261502 (2006).

${ }^{5}$ B. G. Heil, U. Czarnetzki, R. P. Brinkmann, and T. Mussenbrock, J. Phys. D: Appl. Phys. 41, 165202 (2008).

${ }^{6}$ Y. Yang and M. J. Kushner, Plasma Sources Sci. Technol. 19, 055011 (2010).

${ }^{7}$ M. A. Lieberman, J. P. Booth, P. Chabert, J. M. Rax, and M. M. Turner, Plasma Sources Sci. Technol. 11, 283 (2002).

${ }^{8}$ D. Hrunski, F. Mootz, A. Zeuner, A. Janssen, H. Rost, R. Beckmann, S. Binder, E. Schüngel, S. Mohr, D. Luggenhölscher et al., Vacuum 87, 114 (2013).

${ }^{9}$ J. Schulze, T. Gans, D. O'Connell, U. Czarnetzki, A. R. Ellingboe, and M. M. Turner, J. Phys. D: Appl. Phys. 40, 7008 (2007).

${ }^{10}$ D. O'Connell, T. Gans, E. Semmler, and P. Awakowicz, Appl. Phys. Lett. 93, 081502 (2008).

${ }^{11}$ J. Schulze, A. Derzsi, and Z. Donkó, Plasma Sources Sci. Technol. 20, 045008 (2011).

${ }^{12}$ E. Johnson, P. Delattre, and J. Booth, Appl. Phys. Lett. 100, 133504 (2012).

${ }^{13}$ T. Lafleur, P. Delattre, E. Johnson, and J. Booth, Appl. Phys. Lett. 101, 124104 (2012).

${ }^{14}$ S. Mohr, E. Schüngel, J. Schulze, and U. Czarnetzki, J. Phys. D: Appl. Phys. 46, 435201 (2013).

${ }^{15}$ D. J. Coumou, D. H. Clark, T. Kummerer, M. Hopkins, D. Sullivan, and S. Shannon, IEEE Trans. Plasma Sci. 42, 1880 (2014).

${ }^{16}$ J. Waskoenig and T. Gans, Appl. Phys. Lett. 96, 181501 (2010).

${ }^{17}$ C. O'Neill, J. Waskoenig, and T. Gans, Appl. Phys. Lett. 101, 154107 (2012).

${ }^{18}$ A. Greb, K. Niemi, D. O'Connell, and T. Gans, Appl. Phys. Lett. 103, 244101 (2013).

${ }^{19}$ A. Greb, K. Niemi, D. O'Connell, G. J. Ennis, N. MacGearailt, and T. Gans, Phys. Plasmas 20, 053502 (2013).

${ }^{20}$ M. Shibata, N. Nakano, and T. Makabe, J. Appl. Phys. 80, 6142 (1996).

${ }^{21}$ F. X. Bronold, K. Matyash, D. Tskhakaya, R. Schneider, and H. Fehske, J. Phys. D: Appl. Phys. 40, 6583 (2007).

${ }^{22}$ G. J. M. Hagelaar and L. C. Pitchford, Plasma Sources Sci. Technol. 14, 722 (2005).

${ }^{23}$ A. Salabaş and R. P. Brinkmann, Jpn. J. Appl. Phys. 45, 5203 (2006). 